\title{
Interpersonal comparisons of utility for 2 of 3 types of people
}

\author{
R. Duncan Luce
}

Published online: 25 March 2009

(C) The Author(s) 2009. This article is published with open access at Springerlink.com

\begin{abstract}
This article argues that there is a natural solution to carry out interpersonal comparisons of utility when the theory of gambles is supplemented with a group operation of joint receipts. If so, three types of people can exist, and the two types having multiplicative representations of joint receipt have, in contrast to most utility theories, absolute scales of utility. This makes possible, at least in principle, meaningful interpersonal comparisons of utility with desirable properties, thus resolving a long standing philosophical problem and having potentially important implications in economics. Two behavioral criteria are given for the three classes of people. At this point the relative class sizes are unknown.
\end{abstract}

Keywords Interpersonal comparison of utility · Joint receipt · p-Additive utility · Welfare economics · Weighted utility

Both the economic and philosophic literatures have focussed some discussion on the issue of interpersonal comparisons of utility. With some exceptions, the consensus of many seems to be that we do not have fully satisfactory ways to justify such comparisons. Robbins (1938) argued, very discursively, that such comparisons simply cannot be made. Narens and Luce (1983) showed their impossibility in an ordinal context. Hammond (1991) is especially forceful in the position that, using standard utility theories, no such comparisons are possible, whereas Nozick (1985) claimed that they can be made by equating kinks in the utility functions. Nonetheless, in practice, welfare (not utility per se) comparisons are attempted both at the individual level, as in discussions of family compromises, and at the social level in welfare economics without directly invoking utility. Detailed discussions may be found in Harsanyi (1977),

R. D. Luce $(\varangle)$

Institute for Mathematical Behavioral Sciences, University of California, Social Science Plaza, Irvine, CA 92697-5100, USA

e-mail: rdluce@uci.edu 
Elster and J. Roemer (1991), List (2003), and Binmore (2009) who provides a brief but useful summary. The fundamental point is that for ratio and interval scales, there is no principled way to choose what are "equal" units of utility for different people. These difficulties, of course, raise huge theoretical problems for welfare economics that do not seem to have been effectively resolved.

In this article, a new approach is explored which is based on the idea that not only do gambles have representations that involve both addition and multiplication, but also so should the representations of joint receipts which are assumed to interlock with gambles in a fully rational fashion (segregation, (9)). This leads to representations which turn out to have three quite distinct forms. One is additive over joint receipts, which really is the classical case, and the resulting scale is a ratio one for which interpersonal comparisons are impossible. The other two differ in having, instead, multiplicative representations that are absolute scales. For these two sub-cases, I propose a simple hypothesis as to what such an interpersonal comparison means and arrive at a few of its elementary properties.

It should be added that these ideas by no means solve the issues of social welfare comparisons, although they may lay the ground work for a new approach to it. This remains to be seen.

\section{1 p-Additive representations}

Suppose that $X$ is the set of consequences under consideration, that $\succsim$ is a weak (preference) order over $X$, and that $\oplus$ is a binary operation (of joint receipt) on $X$. As usual, $x \sim y$ means both $x \succsim y$ and $y \succsim x$. Because the indifference relation $\sim$ is an equivalence relation, we are in reality working with equivalence classes, for which we assume that $\langle X, e, \oplus, \sim\rangle$ satisfies the usual axioms of an Abelian (weakly commutative) group with identity $e$. Moreover, we assume that $\langle X, e, \oplus, \succsim\rangle$ on equivalence classes is a solvable, Archimedean-ordered, Abelian group with an isomorphism onto the additive real numbers. For the equivalence classes, the operation $\oplus$ is closed, has an identity, is commutative and associative, and each element has an inverse satisfying the usual axioms of a solvable, Archimedean-ordered, Abelian group (Hölder 1901; Krantz et al. 1971, Chaps. 2 and 3).

Examples of joint receipt are ubiquitous, e.g., choosing two commodities, such as steak and a can of soup, at a store. As we are all aware, steak is an uncertain alternative, whereas a can of soup is highly standard and is often treated as without risk or uncertainty. The steak can be imbedded in $X$ by invoking its certainty equivalent. Another familiar example is receiving a check and a bill in the mail. Both examples generalize to any finite size bundles because the operation is associative.

Instead of investigating the representations into the real numbers $\mathbb{R}$ under just addition, $\langle\mathbb{R}, \geq,+\rangle$, as is usually done, let us suppose, as is true of theories for uncertain alternatives that the representations of $\oplus$ are onto suitable (defined below) subintervals of $\langle\mathbb{R}, \geq,+, \times\rangle$ that are closed under addition and multiplication. Under standard assumptions, the only one consistent with Hölder's axioms has the form

$$
U^{*}(x \oplus y)=U^{*}(x)+U^{*}(y)+\delta^{*} U^{*}(x) U^{*}(y)
$$


(Luce 2000, p. 152). The form (1) is called a p-additive (p for polynomial) utility representation because it with $\delta^{*} \neq 0$ is the only polynomial form with $U^{*}(e)=0$ that can be transformed into an additive representation (by the logarithm of (7) below).

Note that for the representation (1) to make dimensional sense when $\delta^{*} \neq 0$, the unit of $\delta^{*}$ must be the unit of $\left(U^{*}\right)^{-1}$, i.e., that $\delta^{*} U^{*}$ is dimensionless in those cases. So we define

$$
\delta:=\operatorname{sgn}\left(\delta^{*}\right)
$$

i.e., the $\operatorname{sign}$ of $\delta^{*}$, and for $\delta \neq 0$

$$
U:=\left|\delta^{*}\right| U^{*}
$$

So, $U$ is dimensionless when $\delta=-1$ or 1 . The notations $U$ and $\delta$ are used from now on.

\subsection{Representations for $\delta=0$ and $\delta \neq 0$}

For the case $\delta=0$, the $U$ representation corresponding to (1) simplifies to the purely additive form

$$
U(x \oplus y)=U(x)+U(y),
$$

and the utility of both the gains and losses are unbounded because $n$ iterations of $x$, denoted $n x$, has $U(n x)=n U(x)$. Moreover, the additive case is of ratio scale type because $e$ is the identity of $\oplus$.

For $\delta \neq 0$, we may rewrite (1) in terms of $U$ as

$$
1+\delta U(x \oplus y)=[1+\delta U(x)][1+\delta U(y)],
$$

where, now, $\delta=-1$ or 1 . $\mathrm{Ng}$ et al. (2009) examine these cases, showing that under the conditions needed to prove results below, for $\delta=1, U$ maps onto $]-\frac{1}{\delta}, \infty[=$ ] $-1, \infty$ [ which simply means that the utility for gains $(x \succsim e)$ is (subjectively) unbounded, whereas the disutility for losses $(x \precsim e)$ is bounded. For $\delta=-1, U$ maps

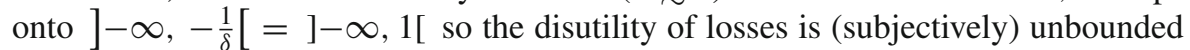
whereas that for gains is bounded.

This means that to the degree that the axioms justifying the representation are satisfied, we may expect people to fall into one of three quite distinct categories with inherently different non-linear forms. (A fourth category with both bounded gains and bounded losses is mentioned in Appendix 5.1.) A major empirical implication of these facts is that it is very unwise to average data from people who have not been pre-screened for, at least, type. This is because when one averages inherently different functions, that average function may well be unlike any of it components (see Fig. 1). Therefore, one needs, at a minimum, to ascertain to which of the three types each person belongs, which is taken up in Sect. 1.3. 

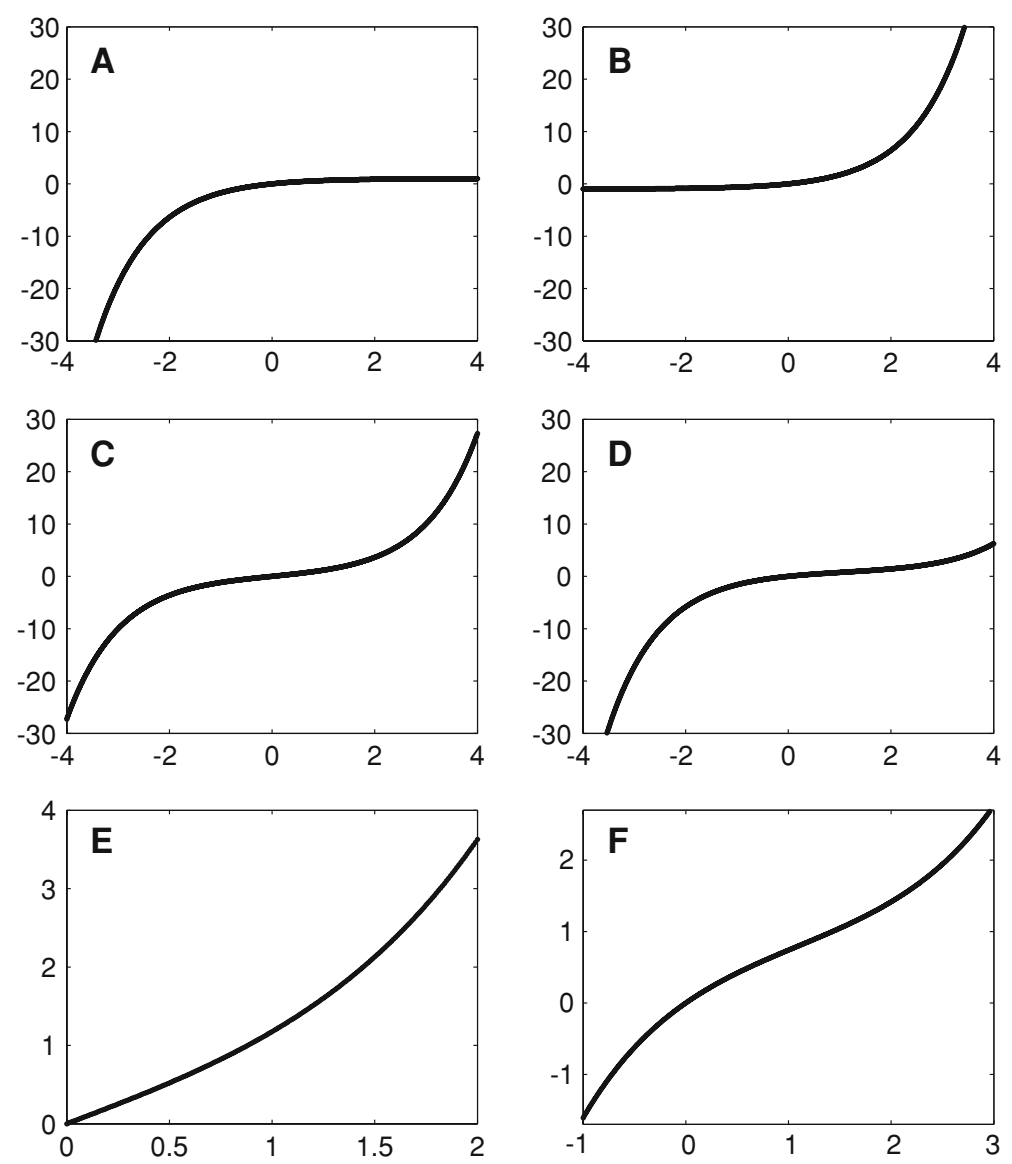

Fig. 1 Panel a $U=1-x^{-\alpha x}(\alpha=1)$; Panel $\mathbf{b} U=\mathrm{e}^{\beta x}-1(\beta=1)$. Panel c Average of Panels $\mathbf{a}$ and $\mathbf{b}$. Panel d 9: 1 average of Panels a and $\mathbf{b}$. Panel e Zoom in on region near $x=0$ of Panel $\mathbf{c}$. Panel $\mathbf{f}$ Zoom in on region near $x=1.1$, the inflexion point, of Panel $\mathbf{d}$

Observe that for $\delta \neq 0$, the transformation

$$
V(x):=1+\delta U(x)
$$

which is referred to as a value representation, is an absolute scale because $U$, and so $\delta U$, is absolute. The $V$ scale maps onto $] 0, \infty[$ in both non-zero cases. The difference being that $V$ is order preserving for $\delta=1$ and order reversing for $\delta=-1$. Moreover, we see that together (5) and (6) yield the multiplicative representation

$$
V(x \oplus y)=V(x) V(y) .
$$

Note that no scale factor $\alpha \neq 1$ maintains the multiplicative representation, (7). In general, such a multiplicative representation is unique only up to an arbitrary power, but as we shall see even that degree of freedom is lost. 


\subsection{Binary uncertain gambles}

Let $x$ and $y$ be consequences from $X$, and let $C$ and $D$ be disjoint chance events arising in a family of chance "experiments." A binary uncertain alternative-often, although somewhat misleadingly, called a gamble, the term which I will use-is based on an experiment whose "universal set" may be partitioned into $C$ and $D$. Each sub-event leads to its own consequence, so that the gamble has two chance branches $(x, C)$ and $(y, D)$. We write it as $(x, C ; y, D)$.

For $x \succsim y$, observe that over the equivalence classes

$$
x \oplus y^{-1}=z \Leftrightarrow x=z \oplus y .
$$

Two key assumptions linking the representations arising from joint receipt and those from the gambling structure are:

Segregation:

$$
\left(x \oplus y^{-1}, C ; e, D\right) \oplus y \sim(x, C ; y, D) .
$$

Segregation seems highly rational: On the left the consequences are $\left(x \oplus y^{-1}\right) \oplus y=x$ if $C$ occurs and $e \oplus y=y$ if $D$ occurs, and the right of course yields exactly the same thing.

Separability: The set of so-called unitary gambles of gains, those of the form $(x, C ; e$, $D$ ), where $x \succsim e$, satisfies a version of the axioms of conjoint structure on $X \times \mathcal{B}$, where $\mathcal{B}$ is the algebra of events. A formal axiomatization was given by Marley and Luce (2002) for gains (and losses), but for present purposes all that is really needed is (10) below.

For the case $\delta=0$, Luce (2000, Theorem 4.4.4) shows that under certain density assumptions, segregation, and separability, that there is a ratio scale utility function $U$ over gains, $x \succsim e$, and a subjective weighting function $S_{C \cup D}$ with the following properties. $U$ and $S_{C \cup D}$ are onto intervals and

$$
U(x, C ; e, D)=U(x) S_{C \cup D}(C) \quad(x \succsim e) .
$$

By segregation one obtains for any $x, y \in X$ with $x \succ y$,

$$
U(x, C ; y, D)=U(x) S_{C \cup D}(C)+U(y)\left[1-S_{C \cup D}(C)\right] .
$$

Assuming that there is a p-additive representation $U^{\prime}$ with $\delta \neq 0$ and a separable representation $\left(U^{\prime \prime}, S_{C \cup D}^{\prime \prime}\right), \mathrm{Ng}$ et al. (2009) drawing upon Luce (2000, Theorem 4.4.6) for gains, show that under the above assumptions there is a unique $U$ that is p-additive and $\left(U, S_{C \cup D}\right)$ is separable, that (11) holds and, with the definition (6) of $V$ also yields, for binary gambles with $x \succ y$,

$$
V(x, C ; y, D)=V(x) S_{C \cup D}(C)+V(y)\left[1-S_{C \cup D}(C)\right] .
$$


Note that if separability (10) holds not just for gains, but for all $x \in X$, then the following stronger property holds also:

Complementarity:

$$
S_{C \cup D}(C)+S_{C \cup D}(D)=1 .
$$

With this property, the rank dependence in (11) and (12) disappears.

\subsection{Behavioral criteria to distinguish $\delta=1,0,-1$}

An obvious question to be addressed is how to tell whether $\delta=1,0$, or -1 . Theorem 4.7, p. 157 of Keeney and Raiffa (1993/1976) addresses a related question but in a much more restricted context. ${ }^{1}$ Part II of the following Proposition was motivated by their discussion (p. 230) of additive independence in risky money gambles defined by

$$
\left(x+x^{\prime}, \frac{1}{2} ; y+y^{\prime}, \frac{1}{2}\right) \sim\left(x+y^{\prime}, \frac{1}{2} ; y+x^{\prime}, \frac{1}{2}\right) \text {. }
$$

That together with comments by Igor Kopylov ${ }^{2}$ and Peter Wakker ${ }^{3}$ led to the following development of Part II of Proposition 2.

It is quite easy to verify in (11) and (12) that if we impose segregation and separability without the condition that $x \succ y$, then both lead to complementarity, (13).

Lemma 1 Suppose that a p-additive representation exists, that both segregation and separability for gains hold, that uncertain binary gambles satisfy (11) for $\delta=0$ and (12) for $\delta \neq 0$, and that complementarity(13) holds. If there exist consequences $x \succ y$ and events $C, D$ such that

$$
(x, C ; y, D) \sim(x, D ; y, C) \text {. }
$$

then

$$
S_{C \cup D}(C)=S_{C \cup D}(D)=\frac{1}{2}
$$

The three assumptions of the Lemma are redundant because, as shown in Luce (2000, Theorem 4.4.4), any two implies the third.

All proofs may be found in Appendix 5.2.

Part I of the following Proposition and its proof are due to C. T. $\mathrm{Ng}$, whom I thank.

Proposition 2 Suppose that a p-additive representation exists, that both segregation and separability for gains hold, that uncertain binary gambles satisfy (11) for $\delta=0$ and (12) for $\delta \neq 0$.

\footnotetext{
1 Brought to my attention by A.A.J. Marley, personal communication, January, 2008.

2 Personal communication, February, 2008.

3 Personal communication, March, 2008.
} 
Part I Then the following two statements are equivalent:

1.

$$
\delta=\left\{\begin{array}{c}
1 \\
0 \\
-1
\end{array}\right\}
$$

2. For all $x, y \in X$, with $x \succ e, y \succ e$, and for all non-trivial event partitions $\{C, D\}$ with independent replications $\left\{C^{\prime}, D^{\prime}\right\}$ and $\left\{C^{\prime \prime}, D^{\prime \prime}\right\}$, then

$$
(x \oplus y, C ; e, D)\left\{\begin{array}{l}
\succ \\
\sim \\
\prec
\end{array}\right\}\left(x, C^{\prime} ; e, D^{\prime}\right) \oplus\left(y, C^{\prime \prime} ; e, D^{\prime \prime}\right)
$$

Part II Suppose, further, that complementarity, (13), holds for the structure and $C$ and D satisfy (15) of Lemma 1, then, for all $x \succ x^{\prime} \succ y \succ y^{\prime}$, the above two conditions are also equivalent to

$$
\left(x \oplus x^{\prime}, C ; y \oplus y^{\prime}, D\right)\left\{\begin{array}{l}
\succ \\
\sim \\
\prec
\end{array}\right\}\left(x \oplus y, C ; x^{\prime} \oplus y^{\prime}, D\right) .
$$

Again, the three assumptions are redundant.

Clearly, the criterion (17) is more general and easier to check empirically than (18) which rests upon finding events that are subjectively $\frac{1}{2}$.

Despite this disadvantage, the criterion (18) does not appear to be unduly difficult to check empirically when dealing with money lotteries with known probabilities, and it has a very simple intuitive interpretation. If for money $x \oplus y=x+y$, as seems quite plausible, then we are speaking of 50:50 bets $\left(x, \frac{1}{2} ; y, \frac{1}{2}\right)$ that have the same expected value but with a larger variance on the left than the right. Often, in the context of choice, these are called, respectively, risky and safe lotteries. To be specific, several examples of Part II of Proposition 2 are

$$
\begin{aligned}
& \left(160, \frac{1}{2} ; 50, \frac{1}{2}\right) \text { versus }\left(140, \frac{1}{2} ; 70, \frac{1}{2}\right) \\
& \left(190, \frac{1}{2} ; 30, \frac{1}{2}\right) \text { versus }\left(120, \frac{1}{2} ; 100, \frac{1}{2}\right) \\
& \left(1900, \frac{1}{2} ; 60, \frac{1}{2}\right) \text { versus }\left(1050, \frac{1}{2} ; 910, \frac{1}{2}\right) .
\end{aligned}
$$

Do you feel $\prec, \sim, \succ$ for each? I, for one, am clearly a $\delta=-1(\prec)$ type for money gambles. My guess is that people who gamble a good deal are of type $\delta=1$. Are there any who are of type $\delta=0$ ? If not, then classical utility theory is not descriptive. My guess is that these types may be domain specific: athletics, including risky ones such as skiing and mountain climbing, may differ from finances, or from cuisine adventures. 
It is clearly important to examine one of the criteria of Proposition 2 carefully for fairly large and varied populations to estimate, in particular, first, whether the $\delta=0$ class is non-empty and, second, the relative sizes of the $\delta=1$ and -1 subpopulations. Very informal polling at three presentations of these results ${ }^{4}$ suggest that most academics are financially $\delta=-1$, a few are $\delta=1$, and a very few, if any, $\delta=0$. Serious data are needed.

\section{Interpersonal comparisons of utility (IPC)}

Next, an hypothesis is offered concerning interpersonal comparisons of utility for the cases with $\delta \neq 0$. Until the experiments implicit in Proposition 2 are carried out, there can be no certainty about just how large a class of people this covers.

But first, let us consider what properties we expect a potential concept of IPC to exhibit. We list possible demands.

\subsection{Demands on a definition of IPC}

Let $k, l$, and $m$ be three different people and let $x_{(k)} \approx y_{(l)}$ mean that consequence $x$ for person $k$ has the "same utility" as consequence $y$ for person $l$. The minimal properties that many feel such an IPC relation should satisfy are:

(i) Reflexivity:

$$
\forall x \in X, x_{(k)} \approx x_{(k)} .
$$

(ii) Symmetry:

$$
\forall x, y \in X, x_{(k)} \approx y_{(l)} \Leftrightarrow y_{(l)} \approx x_{(k)} .
$$

(iii) Transitivity: If $\delta_{l} \neq 0, \delta_{k} \neq 0$, and $\delta_{m} \neq 0$, then for $\forall x, y, z \in X$ :

$$
\text { If } x_{(k)} \approx y_{(l)} \text { and } y_{(l)} \approx z_{(m)} \text {, then } x_{(k)} \approx z_{(m)} \text {. }
$$

Transitivity simply asserts that if one knows that $(x, y)$ is a matching pair between $k$ and $l$ and that $(y, z)$ is a match between $l$ and $m$, then by transitivity one knows that $(x, z)$ is a match between $k$ and $m$.

Any $\approx$ satisfying (i-iii) is, of course, an equivalence relation.

A rather more controversial condition is whether we should expect:

(iv) Invariance under joint receipt:

$$
\text { If } x_{(k)} \approx y_{(l)} \text { and } x_{(k)}^{\prime} \approx y_{(l)}^{\prime}, \text { then }\left(x \oplus x^{\prime}\right)_{(k)} \approx\left(y \oplus y^{\prime}\right)_{(l)} .
$$

\footnotetext{
4 UC Irvine, May 8, 2008; FUR, Barcelona, July 3, 2008; and Edwards Bayes Conference, Fullerton, CA, January 8, 2009.
} 
The operation $\oplus$ is not associated with the individual because joint receipt is a purely objective matter.

\subsection{A possible definition of IPC for $\delta \neq 0$}

In the non-additive cases, suppose that we define interpersonal comparability (IPC) by

$$
x_{(k)} \approx y_{(l)} \Leftrightarrow U_{k}(x)=U_{l}(y) .
$$

This is well defined because with $\delta \neq 0, U_{k}$ and $U_{l}$ are absolute scales and so no ambiguity arises in equating them. This contrasts with the unit ambiguity in the case of interval or ratio scales. Of course, in practice it will not be easy to establish $\approx$ because it is necessary to estimate $U_{k}$ and $U_{l}$, which, although possible in principle, can often be difficult to do in practice. The key issue is establishing for any utility estimate using standard methods exactly the value of the asymptote $( \pm 1)$, which can then be used to normalize that function.

As will be shown, the equivalence relation property of $\approx$ follows fairly readily. But, in general, we will not be able to satisfy invariance under joint receipt. Intuitively, the reason is that the utility of two things simply need not "add up" in the same way for different types of people, so in general $U_{k}\left(x \oplus x^{\prime}\right) \neq U_{l}\left(y \oplus y^{\prime}\right)$ when $U_{k}(x)=U_{l}(y)$ and $U_{k}\left(x^{\prime}\right)=U_{l}\left(y^{\prime}\right)$. For example (see Sect. 3), suppose that the domain $X$ is amounts of money, that $x \oplus y=x+y$, and that there is a constant $\alpha_{k}>0$ such that $U_{k}(x)=\alpha_{k} x$ and $\alpha_{l}>0$ such that $U_{l}(x)=1-\mathrm{e}^{\alpha_{l} x}$. So $U_{k}$ is additive and $U_{l}$ is p-additive with $\delta=-1$. Choose $x, x^{\prime}, y$ and $y^{\prime}$ such that

$$
\begin{aligned}
U_{k}(x) & =U_{l}(y) \Leftrightarrow \alpha_{k} x=1-\mathrm{e}^{\alpha_{l} y} \\
U_{k}\left(x^{\prime}\right) & =U_{l}\left(y^{\prime}\right) \Leftrightarrow \alpha_{k} x^{\prime}=1-\mathrm{e}^{\alpha_{l} y^{\prime}},
\end{aligned}
$$

then

$$
U_{k}\left(x \oplus x^{\prime}\right)=U_{k}\left(x+x^{\prime}\right)=\alpha_{k}\left(x+x^{\prime}\right)
$$

and

$$
\begin{aligned}
U_{l}\left(y \oplus y^{\prime}\right) & =U_{l}\left(y+y^{\prime}\right)=1-\mathrm{e}^{\alpha_{l}\left(y+y^{\prime}\right)} \\
& \neq \alpha_{k}\left(x+x^{\prime}\right)=U_{k}\left(x \oplus x^{\prime}\right) .
\end{aligned}
$$

There is nothing special about the choice of these $U$ functions except that they are not equal and are contained within the families discussed in Proposition 4 and its Corollary.

Assuming that IPC is given by (23), we explore the above four properties. 


\subsection{Two $\delta \neq 0$ people}

Suppose, first, that persons $k$ and $l$ are of the same type, i.e., have either $\delta_{k}=\delta_{l}=1$ or $\delta_{k}=\delta_{l}=-1$. In these cases, the multiplicative representations $V_{k}$ and $V_{l}$ are absolute scales and so (23) is equivalent to

$$
x_{(k)} \approx y_{(l)} \Leftrightarrow V_{k}(x)=V_{l}(y) .
$$

Next, suppose that they are of different type, e.g., $\delta_{k}=1$ and $\delta_{l}=-1$. Then, matches can only arise in the common region where both utility functions exist, namely,

$$
\mathcal{D}_{-1,1}=\left\{\left(x_{(k)}, y_{(l)}\right) \mid U_{k}(x)=U_{l}(y) \in\right]-1,1[\} .
$$

Thus, for $\left(x_{(k)}, y_{(l)}\right) \in \mathcal{D}_{-1,1}$ we have $U_{k}(x)=U_{l}(y)$ and so, using (6) twice,

$$
V_{k}(x)-1=U_{k}(x)=U_{l}(y)=1-V_{l}(y) .
$$

So, from (23) and (25),

$$
x_{(k)} \approx y_{(l)} \Leftrightarrow V_{k}(x)-1=1-V_{l}(y) .
$$

Proposition 3 Suppose that a p-additive representation with $\delta \neq 0$ exists for each person, that both segregation and separability over gains hold, that uncertain binary gambles satisfy (12).

I. If two individuals $l$ and $k$ are both $p$-additive with $\delta_{l}=\delta_{k}=1$ or -1 , then $\approx$ defined by (23) is an equivalence relation and is invariant under joint receipt.

II. If person $k$ is of type $1, l$ is of type -1 , and $m$ is of either type, then for $(x, y) \in$ $\mathcal{D}_{-1,1}$ the relation $\approx$ is an equivalence one, but it is not necessarily invariant under joint receipt.

The above formulations have been for pure consequences, but that extends to gambles $f$ via the use of certainty equivalents, i.e., for a gamble $f$ there is a $C E(f) \in X$ such that $f \sim C E(f)$.

\section{Utility for money}

\subsection{General representations}

This section is in response to questions raised in person by Igor Kopylov and Stergios Skaperdas: what amount of money must a wealthier person gain or lose so it has equal utility to that of a poorer person for gaining or losing, say, a dollar. The answer is provided at the end of the section. To this end, we explore the special case of the utility of increments of money. This we take to mean that for any $x, y \in \mathbb{R}, \oplus$ is a group operator with identity 0 , and $U$ is strictly increasing. 
Proposition 4 Suppose that the assumptions of Proposition 3 are satisfied when $X=$ $\mathbb{R}$ is money amounts. Then there exists a strictly increasing function $g$ that is additive over $\oplus$ such that:

(i) If $\delta=0$, then $U$ is strictly increasing and onto $\mathbb{R}$ and

$$
U(x)=\alpha g(x) \quad(\alpha>0) .
$$

(ii) If $\delta=1$, then $V=1+U$ is strictly increasing, onto $\mathbb{R}_{+}$, multiplicative, (7), and

$$
U(x)=\mathrm{e}^{\alpha g(x)}-1 \quad(\alpha>0)
$$

(iii) If $\delta=-1$, then $V=1-U$ is strictly decreasing, onto $\mathbb{R}_{+}$, multiplicative, and

$$
U(x)=1-\mathrm{e}^{-\alpha g(x)} \quad(\alpha>0) .
$$

We next consider a special case which many economists seem to believe should hold for money, at least for rational people, namely, that money joint receipt is just addition:

$$
x \oplus y=x+y .
$$

Corollary 5 Under the conditions of Proposition 4, for $x \in \mathbb{R}$, (30) is equivalent to

$$
g(x)=x,
$$

and so:

(i) If $\delta=0$, then

$$
U(x)=\alpha x \quad(\alpha>0) .
$$

(ii) If $\delta=1$, then

$$
U(x)=e^{\alpha x}-1 \quad(\alpha>0)
$$

(iii) If $\delta=-1$, then

$$
U(x)=1-e^{-\alpha x} \quad(\alpha>0) .
$$

Note that for $\delta=1$, where we have (33), it is well known that $U$ is strictly increasing and convex, which many identify as corresponding to risk seeking behavior.

And for $\delta=-1$, it follows from the first and second derivatives that $U$ is strictly increasing and concave, which corresponds to risk averse behavior. 
Assuming the functions (33) and (34) of the Corollary, let us examine what happens when we have a weighted average of proportion $a$ of type $\delta=-1$ and $1-a$ of type $\delta=1$, with the same parameter for all people of the same type. Thus, the average is

$$
\bar{U}(x)=a\left(1-\mathrm{e}^{-\alpha x}\right)+(1-a)\left(\mathrm{e}^{\beta x}-1\right) .
$$

Note that

$$
\begin{gathered}
\bar{U}^{\prime \prime}(x)=-a \alpha^{2} \mathrm{e}^{-\alpha x}+(1-a) \beta^{2} \mathrm{e}^{\beta x} \gtrless 0 \\
\Leftrightarrow x \gtrless \frac{1}{\alpha+\beta}\left(\frac{\alpha}{\beta}\right)^{2} \ln \left(\frac{a}{1-a}\right) .
\end{gathered}
$$

For the special case $\alpha=\beta$ this yields

$$
x \gtrless\left\{\begin{array}{lrl}
0, & a & =\frac{1}{2} \\
\frac{1}{2} \ln 9 \approx 1.1, a & =0.9
\end{array} .\right.
$$

Observe that $\alpha x$ must be dimensionless, and so to have the amounts of money that are likely to be typical, the abscissa's must be multiplied by $10^{n}$, where $n$ is at least 6 and $\alpha$ by the reciprocal factor. Figure 1 shows for $\alpha=\beta=1$ plots for $\delta=-1$ (Panel a) and for $\delta=1$. The second and third rows are the averages with $a=\frac{1}{2}$ and 0.9 cases on the left and right, respectively. Row 3 focuses in on the regions where the curvature changes. One readily sees just how misleading such average data can be.

A major open problem is how, from the data that we can collect from an individual, do we go about estimating what amounts to the money amount corresponding closely to the asymptote of the $\delta \neq 0$ functions. This means, developing methods to estimate $\alpha$ in Corollary 5 or the expression $\alpha g(x)$ in Proposition 4. Inroads on this kind of problem have been developed by Abdellaoui et al. (2007).

\subsection{Interpersonal comparisons for exponential utility functions}

Suppose that the comparison is between two people of type $\delta=1$ with exponential utility and constants $\alpha_{k}, \alpha_{l}$. Then

$$
x_{(k)} \approx y_{(l)} \Leftrightarrow \mathrm{e}^{\alpha_{k} x}-1=\mathrm{e}^{\alpha_{l} y}-1 \Leftrightarrow \frac{y}{x}=\frac{\alpha_{k}}{\alpha_{l}} .
$$

If we conjecture, as seems plausible, that when person $l$ is wealthier than person $k$, then a larger increment, $y$, is needed to match in utility the increment $x$ for a poorer person, i.e., $y>x \Leftrightarrow \alpha_{l}<\alpha_{k}$. Exactly what "wealthier than" means does not matter beyond that $x_{(k)} \approx y_{(l)}$ holds only for $y>x$, i.e., that it takes a larger money increment $y$ to give the wealthier person the same satisfaction, as measured by $U_{l}$, as the increment $x$ give the relatively poorer person, as measured by $U_{k}$. By the transitivity 
of $\approx$, increasing wealth corresponds to decreasing the exponent in the exponential utility form.

Similarly, for two people of type $\delta=-1$,

$$
x_{(k)} \approx y_{(l)} \Leftrightarrow \frac{y}{x}=\frac{\alpha_{k}}{\alpha_{l}} .
$$

Finally, for a person 1 of type $\delta=1$ and person 2 of type $\delta=-1$, we obtain for

$$
-\infty<x<\frac{\ln 2}{\alpha_{k}} \text { and } \frac{-\ln 2}{\alpha_{l}}<y<\infty,
$$

which are needed to keep the utilities of the two amounts within the prescribed interval ]$-1,1[$, then

$$
x_{(k)} \approx y_{(l)} \Leftrightarrow y=\frac{-\ln \left(2-\mathrm{e}^{\alpha_{k} x}\right)}{\alpha_{l}} .
$$

\section{Conclusions}

In principle, one can use the behavioral criteria provided by Proposition 2 to decide, for any person, into which category, $\delta=1,0$, or -1 , he or she falls. The criterion of Part II works easily for experiments with given probabilities and money consequences, i.e., lotteries. For general events and consequences, Part I provides a simple criterion to test without having to estimate any subjective probabilities.

Proposition 2, Part 2, suggests, to me at least, that the class of $\delta=0$ people-those who perceive utility as unbounded for both gains and for losses-may be, in fact, empty or close to it. This bears empirical investigation.

For the $\delta \neq 0$ cases, there are two disjoint classes of people corresponding to $\delta=1$ or $\delta=-1$. It seems intuitive to me, although this is certainly speculative, that whether a person exhibits unbounded gains and bounded losses $(\delta=1)$ or bounded gains and unbounded losses $(\delta=-1)$ correspond, respectively, to the ideas of risk seeking and risk averse people. People for which $\delta=0$ can be described as risk neutral.

Interestingly, within the framework of $\oplus$ having a $p$-additive representation, the case where utility is both bounded for gains and bounded for losses simply does not arise. A partial extension to that case is outlined in Appendix 5.1.

For the $\delta \neq 0$ case, an hypothesis was formulated as to what "interpersonal comparison of utility" might mean, and several of its elementary properties were derived. These are embodied in Proposition 3. I do not see any comparable solution for the $\delta=0$ subpopulation, if such people exist, because the utility function is a ratio scale, not an absolute one.

These results were detailed for money consequences.

Three major open problems were mentioned above: (1) what is a practical method for estimating the asymptote of utility in the $\delta \neq 0$ cases? (2) how well are the money data for individuals fit by functions of Corollary 5, in particular, assuming (30) holds 
separately for gains and for losses? (3) can a sensible concept of social welfare be formulated using the absolute scales that arise when $\delta \neq 0$ ?

A fourth open problem is raised in Appendix 5.1, namely, to develop a behavioral theory of bounded mixed gains and losses.

Acknowledgments This work was supported in part by National Science Foundation grant SES-0452756 to the University of California, Irvine. Any opinions, findings, and conclusions or recommendations expressed in this material are those of the author and do not necessarily reflect the views of the National Science Foundation. The author has benefited considerably from discussions with, and suggestions on earlier versions of this article, by János Aczél, Ken Binmore, Han Bleichrodt (who caught errors in an earlier version concerning constraints in the first Proposition), Jean-Claude Falmagne (who spotted ambiguities), Igor Kopylov, A. A. J. Marley (who, in his usual way, has provided very detailed and useful comments), C. T. Ng (who not only spotted ambiguities but provided Part I of Proposition 2), Drazen Prelec, Donald Saari, Stergios Skaperdas, and Peter Wakker (whose detailed suggestions may have increased its accessibility and clarity).

Open Access This article is distributed under the terms of the Creative Commons Attribution Noncommercial License which permits any noncommercial use, distribution, and reproduction in any medium, provided the original author(s) and source are credited.

\section{Appendices}

\subsection{Outline of a generalization to four classes of $\delta \neq 0$ people}

Had we taken the approach of Luce (2000, Chaps. 6 and 7), we would have worked not with the full group $\langle X, \oplus, e, \succsim\rangle$ but separately with the two Abelian semigroups $\left\langle X^{(+)}, \oplus, e, \succsim\right\rangle$ and $\left\langle X^{(-)}, \oplus, e, \succsim\right\rangle$ where

$$
X^{(+)}:=\{x \in X \mid x \succsim e\}, \quad X^{(-)}:=\{x \in X \mid x \precsim e\}
$$

The arguments about absolute scales for $\delta \neq 0$ continue to hold for these two subspaces. This means that, in principle, there are four cases to be explored:

$$
\left(\delta^{(+)}, \delta^{(-)}\right)=(1,1),(1,-1),(-1,1),(-1,-1)
$$

In the main body of the article, I considered only the first and fourth and for money I assumed the same proportionality constants for gains and losses. In this generalization, we admit different proportionality constants and, in addition, we consider two the mixed cases: $(1,-1)$, which is unbounded in both directions, and $(-1,1)$, which is bounded from both above and below.

The fitting of several models, being conducted by Han Bleichrodt, to data from mid-level executives collected by him, Mohammed Abdellaoui, and Hilda Kammoun was the major motivation for considering these generalizations. Those results will be reported elsewhere.

The major issue in developing a complete theory of this type is how to handle mixed gains and losses, which preoccupied me, not fully successfully, in Luce (2000, Chaps. 6 and 7). 


\subsection{Proofs}

\subsubsection{Lemma 3}

Proof Suppose that $x \succ y$ and that $C, D$ are such that

$$
(x, C ; y, D) \sim(x, D ; y, C) .
$$

By segregation, this becomes when $\delta=0$

$$
\begin{aligned}
\left(x \oplus y^{-1}, C ; e, D\right) & \sim\left(x \oplus y^{-1}, D ; e, C\right) \\
\Leftrightarrow U\left(x \oplus y^{-1}, C ; e, D\right) & =U\left(x \oplus y^{-1}, D ; e, C\right)
\end{aligned}
$$

By separability,

$$
\begin{aligned}
U\left(x \oplus y^{-1}\right) S_{C \cup D}(C) & =U\left(x \oplus y^{-1}\right) S_{C \cup D}(D) \\
\Leftrightarrow S_{C \cup D}(C) & =S_{C \cup D}(D),
\end{aligned}
$$

and adding complementarity (13) yields (15). For $\delta \neq 0$, the parallel argument involves $U$ replaced by $V$. The conclusion is not altered.

\subsubsection{Proposition 2}

Proof Part I We begin by assuming that I.1 holds and prove I.2. This proof is due to C. T. Ng. First, we consider the case where $x, y$ are either both gains or both losses. Thus, in both cases $U(x) U(y)>0$.

Case (i) Suppose that $\delta=1$. Then keeping in mind that $x \succ e, y \succ e$ and that independent replications of $\{C, D\}$ means $S_{C \cup D}(C)=S_{C^{\prime} \cup D^{\prime}}\left(C^{\prime}\right)=S_{C^{\prime \prime} \cup D^{\prime \prime}}\left(C^{\prime \prime}\right)$,

$$
\begin{aligned}
1+ & U(x \oplus y, C ; e, D) \\
= & 1+U(x \oplus y) S_{C \cup D}(C) \\
= & 1+[U(x)+U(y)+U(x) U(y)] S_{C \cup D}(C) \\
= & 1+U(x) S_{C \cup D}(C)+U(y) S_{C \cup D}(C)+U(x) U(y) S_{C \cup D}(C) \\
> & 1+U(x) S_{C \cup D}(C)+U(y) S_{C \cup D}(C)+U(x) U(y) S_{C \cup D}(C) S_{C \cup D}(C) \\
= & 1+U(x) S_{C^{\prime} \cup D^{\prime}}\left(C^{\prime}\right)+U(y) S_{C^{\prime \prime} \cup D^{\prime \prime}}\left(C^{\prime \prime}\right) \\
& +U(x) U(y) S_{C^{\prime} \cup D^{\prime}}\left(C^{\prime}\right) S_{C^{\prime \prime} \cup D^{\prime \prime}}\left(C^{\prime \prime}\right) \\
= & {\left[1+U(x) S_{C^{\prime} \cup D^{\prime}}\left(C^{\prime}\right)\right]\left[1+U(y) S_{C^{\prime \prime} \cup D^{\prime \prime}}\left(C^{\prime \prime}\right)\right] } \\
= & {\left[1+U\left(x, C^{\prime} ; e, D^{\prime}\right)\right]\left[1+U\left(y, C^{\prime \prime} ; e, D^{\prime \prime}\right)\right] } \\
= & V\left(x, C^{\prime} ; e, D^{\prime}\right) V\left(y, C^{\prime \prime} ; e, D^{\prime \prime}\right) \\
= & V\left(\left(x, C^{\prime} ; e, D^{\prime}\right) \oplus\left(y, C^{\prime \prime} ; e, D^{\prime \prime}\right)\right) \\
= & 1+U\left(\left(x, C^{\prime} ; e, D^{\prime}\right) \oplus\left(y, C^{\prime \prime} ; e, D^{\prime \prime}\right)\right) .
\end{aligned}
$$


So, $U(x \oplus y, C ; e, D)>U\left(\left(x, C^{\prime} ; e, D^{\prime}\right) \oplus\left(y, C^{\prime \prime} ; e, D^{\prime \prime}\right)\right)$ and therefore

$$
(x \oplus y, C ; e, D) \succ\left(x, C^{\prime} ; e, D^{\prime}\right) \oplus\left(y, C^{\prime \prime} ; e, D^{\prime \prime}\right) .
$$

Case (ii) For $\delta=0$, then

$$
\begin{aligned}
U & (x \oplus y, C ; e, D) \\
& =U(x \oplus y) S_{C \cup D}(C) \\
& =[U(x)+U(y)] S_{C \cup D}(C) \\
& =U(x) S_{C^{\prime} \cup D^{\prime}}\left(C^{\prime}\right)+U(y) S_{C^{\prime \prime} \cup D^{\prime \prime}}\left(C^{\prime \prime}\right) \\
& =U\left(x, C^{\prime} ; e, D^{\prime}\right)+U\left(y, C^{\prime \prime} ; e, D^{\prime \prime}\right) \\
& =U\left(\left(x, C^{\prime} ; e, D^{\prime}\right) \oplus\left(y, C^{\prime \prime} ; e, D^{\prime \prime}\right)\right) .
\end{aligned}
$$

This proves

$$
(x \oplus y, C ; e, D) \sim\left(x, C^{\prime} ; e, D^{\prime}\right) \oplus\left(y, C^{\prime \prime} ; e, D^{\prime \prime}\right) .
$$

Case (iii) Suppose that $\delta=-1$. Then using the fact that $U(x) U(y)>0$ because both are gains or both are losses,

$$
\begin{aligned}
1 & -U(x \oplus y, C ; e, D) \\
& =1-U(x \oplus y) S_{C \cup D}(C) \\
& =1-[U(x)+U(y)-U(x) U(y)] S_{C \cup D}(C) \\
& =1-U(x) S_{C \cup D}(C)-U(y) S_{C \cup D}(C)+U(x) U(y) S_{C \cup D}(C) \\
& >1-U(x) S_{C \cup D}(C)-U(y) S_{C \cup D}(C)+U(x) U(y) S_{C \cup D}(C) S_{C \cup D}(C) \\
& =1-U(x) S_{C^{\prime} \cup D^{\prime}}\left(C^{\prime}\right)-U(y) S_{C^{\prime \prime} \cup D^{\prime \prime}}\left(C^{\prime \prime}\right)+U(x) U(y) S_{C^{\prime} \cup D^{\prime}}\left(C^{\prime}\right) S_{C^{\prime \prime} \cup D^{\prime \prime}}\left(C^{\prime \prime}\right) \\
& =\left[1-U(x) S_{C^{\prime} \cup D^{\prime}}\left(C^{\prime}\right)\right]\left[1-U(y) S_{C^{\prime \prime} \cup D^{\prime \prime}}\left(C^{\prime \prime}\right)\right] \\
& =\left[1-U\left(x, C^{\prime} ; e, D^{\prime}\right)\right]\left[1-U\left(y, C^{\prime \prime} ; e, D^{\prime \prime}\right)\right] \\
& =V\left(x, C^{\prime} ; e, D^{\prime}\right) V\left(y, C^{\prime \prime} ; e, D^{\prime \prime}\right) \\
& =V\left(\left(x, C^{\prime} ; e, D^{\prime}\right) \oplus\left(y, C^{\prime \prime} ; e, D^{\prime \prime}\right)\right) \\
& =1-U\left(\left(x, C^{\prime} ; e, D^{\prime}\right) \oplus\left(y, C^{\prime \prime} ; e, D^{\prime \prime}\right)\right) .
\end{aligned}
$$

So, $U(x \oplus y, C ; e, D)<U((x, C ; e, D) \oplus(y, C ; e, D))$ and therefore

$$
(x \oplus y, C ; e, D) \prec\left(x, C^{\prime} ; e, D^{\prime}\right) \oplus\left(y, C^{\prime} ; e, D^{\prime}\right) .
$$

So Part I.1 implies Part I.2.

Part II We now assume that the structure satisfies complementarity, (13). Clearly, this does not affect the equivalence of Parts I.1 and I.2. So, assuming I.1, consider any consequences for which $x \succ x^{\prime} \succ y^{\prime} \succ y$. 
For $\delta=0$,

$$
\begin{aligned}
(x & \left.\oplus x^{\prime}, C ; y \oplus y^{\prime}, D\right) \sim\left(x \oplus y, C ; x^{\prime} \oplus y^{\prime}, D\right) \\
\Leftrightarrow & {\left[\left(U(x)+U\left(x^{\prime}\right)-(U(x)+U(y))\right] \frac{1}{2}\right.} \\
& +\left[\left(U(y)+U\left(y^{\prime}\right)-\left(U\left(x^{\prime}\right)+U\left(y^{\prime}\right)\right)\right] \frac{1}{2}=0\right. \\
& \Leftrightarrow\left[U\left(x^{\prime}\right)-U(y)\right] \frac{1}{2}+\left[U(y)-U\left(x^{\prime}\right)\right] \frac{1}{2}=0 \\
\Leftrightarrow & {\left[U\left(x^{\prime}\right)-U(y)\right] 0=0 . }
\end{aligned}
$$

For $\delta \neq 0$, then when $\delta=1(-1) V$ is order preserving (reversing). Then using (15) and $V(e)=1$

$$
\begin{gathered}
x \succ y^{\prime} \\
\Leftrightarrow V(x)>(<) V\left(y^{\prime}\right) .
\end{gathered}
$$

Because $x^{\prime} \succ y$, we may multiply by $V\left(x^{\prime}\right)-V(y)>(<) 0$ to get,

$$
\begin{aligned}
& V(x)\left[V\left(x^{\prime}\right)-V(y)\right]>V\left(y^{\prime}\right)\left[V\left(x^{\prime}\right)-V(y)\right] \\
& \Leftrightarrow V(x) V\left(x^{\prime}\right)+V(y) V\left(y^{\prime}\right)>V(x) V(y)+V\left(x^{\prime}\right) V\left(y^{\prime}\right) \\
& \Leftrightarrow V\left(x \oplus x^{\prime}\right)+V\left(y \oplus y^{\prime}\right)>V(x \oplus y)+V\left(x^{\prime} \oplus y^{\prime}\right) \\
& \Leftrightarrow V\left(x \oplus x^{\prime}\right) \frac{1}{2}+V\left(y \oplus y^{\prime}\right) \frac{1}{2}>V(x \oplus y) \frac{1}{2}+V\left(x^{\prime} \oplus y^{\prime}\right) \frac{1}{2} \\
& \Leftrightarrow V\left(x \oplus x^{\prime}, C ; y \oplus y^{\prime}, D\right)>V\left(x \oplus y, C ; x^{\prime} \oplus y^{\prime}, D\right) \\
& \Leftrightarrow\left(x \oplus x^{\prime}, C ; y \oplus y^{\prime}, D\right) \succ(\prec)\left(x \oplus y, C ; x^{\prime} \oplus y^{\prime}, D\right),
\end{aligned}
$$

which is the criterion (18) for $\delta \neq 0$.

The converses that Parts $I .2$ and $I I$ each imply Part $I .1$ are obvious because the cases are exclusive and exhaustive.

\subsubsection{Proposition 3}

\section{Proof Part I}

(i) Reflexivity is trivial.

(ii) Symmetry is immediate from the definition.

(iii) Transitivity:

$$
\begin{aligned}
x_{(k)} & \approx y_{(l)} \quad \& \quad y_{(l)} \approx z_{(m)} \\
& \Leftrightarrow V_{k}(x)=V_{l}(y) \quad \& \quad V_{l}(y)=V_{m}(z) \\
& \Rightarrow V_{k}(x)=V_{m}(z) \\
& \Leftrightarrow x_{(l)} \approx z_{(m)} .
\end{aligned}
$$


(iv) Invariance under joint receipt:

$$
\begin{aligned}
x_{(k)} & \approx y_{(l)} \quad \& \quad x_{(k)}^{\prime} \approx y_{(l)}^{\prime} \Rightarrow V_{k}(x)=V_{l}(y) \quad \& \quad V_{k}\left(x^{\prime}\right)=V_{l}\left(y^{\prime}\right) \\
& \Rightarrow V_{k}(x) V_{k}\left(x^{\prime}\right)=V_{l}(y) V_{l}\left(y^{\prime}\right) \Rightarrow V_{k}\left(x \oplus x^{\prime}\right)=V_{l}\left(y \oplus y^{\prime}\right) \\
& \Rightarrow\left(x \oplus x^{\prime}\right)_{(k)} \approx\left(y \oplus y^{\prime}\right)_{(l)} .
\end{aligned}
$$

\section{Part II}

(i) Reflexivity is trivial.

(ii) Symmetry:

$$
x_{(k)} \approx y_{(l)} \Leftrightarrow U_{k}(x)=U_{l}(y) \Leftrightarrow U_{l}(y)=U_{k}(x) \Leftrightarrow y_{(l)} \approx x_{(k)} .
$$

(iii) Transitivity: There are two cases to consider:

Case a: $\delta_{m}=1$, then

$$
\begin{aligned}
x_{(k)} & \approx y_{(l)} \Leftrightarrow V_{k}(x)-1=1-V_{l}(y) \text { and } \\
y_{(l)} & \approx z_{(m)} \Leftrightarrow z_{(m)} \approx y_{(l)} \Leftrightarrow V_{m}(z)-1=1-V_{l}(y) \\
& \Rightarrow V_{k}(x)-1=V_{m}(z)-1 \\
& \Leftrightarrow x_{(k)} \approx z_{(m)},
\end{aligned}
$$

which is transitivity.

Case b: $\delta_{m}=-1$, then using (25)

$$
\begin{aligned}
x_{(k)} & \approx y_{(l)} \Leftrightarrow V_{k}(x)-1=1-V_{l}(y) \text { and } \\
y_{(l)} & \approx z_{(m)} \Leftrightarrow V_{l}(y)=V_{m}(z) \\
& \Rightarrow V_{k}(x)-1=1-V_{m}(z) \\
& \Leftrightarrow x_{(k)} \approx z_{(m)},
\end{aligned}
$$

(iv) If

which again is transitivity.

$$
x_{(k)} \approx y_{(l)} \text { and } x_{(k)}^{\prime} \approx y_{(l)}^{\prime},
$$

IPC means

$$
\begin{aligned}
U_{k}(x) & =U_{l}(y) \text { and } U_{k}\left(x^{\prime}\right)=U_{l}\left(y^{\prime}\right) \\
& \Leftrightarrow V_{k}(x)-1=1-V_{l}(y) \text { and } V_{k}\left(x^{\prime}\right)-1=1-V_{l}\left(y^{\prime}\right) \\
& \Leftrightarrow V_{k}(x)=2-V_{l}(y) \text { and } V_{k}\left(x^{\prime}\right)=2-V_{l}\left(y^{\prime}\right) \\
& \Rightarrow V_{k}\left(x \oplus x^{\prime}\right)=V_{k}(x) V_{k}\left(x^{\prime}\right) \\
& =\left[2-V_{l}(y)\right]\left[2-V_{l}\left(y^{\prime}\right)\right] \\
& \Leftrightarrow V_{k}\left(x \oplus x^{\prime}\right)=4-2 V_{l}(y)-2 V_{l}\left(y^{\prime}\right)+V_{l}\left(y \oplus y^{\prime}\right) \\
& \Leftrightarrow V_{k}\left(x \oplus x^{\prime}\right)-1=3-2 V_{l}(y)-2 V_{l}\left(y^{\prime}\right)+V_{l}\left(y \oplus y^{\prime}\right) \\
& \neq 1-V_{l}\left(y \oplus y^{\prime}\right) .
\end{aligned}
$$


Thus, invariance under joint receipt, $x_{(k)} \oplus x_{(k)}^{\prime} \approx y_{(l)} \oplus y_{(l)}^{\prime}$, does not follow in the mixed case.

\subsubsection{Proposition 4}

Proof (i) $\delta=0$ means that the representation is additive, (4), which we repeat as

$$
U(x \oplus y)=U(x)+U(y)
$$

Aczél (1966, Theorem 3, p. 62) yields the general solutions to a somewhat more general equation than (39). Indeed, in his notation that case becomes: $\oplus$ has a representation of the general form

$$
x \oplus y=g^{-1}[g(x)+g(y)]
$$

where $g$ is strictly increasing and so (27) is obvious.

(ii) $\delta=1$ means that $V=1+U$ which has the multiplicative form (7) which, in turn, is equivalent to

$$
\ln V(x \oplus y)=\ln V(x)+\ln V(y)
$$

This with (27) yields the result.

(iii) $\delta=-1$ means that $V=1-U$ which is equivalent to

$$
\Leftrightarrow \ln V(x \oplus y)=\ln V(x)+\ln V(y) .
$$

Taking into account that $V$ is strictly decreasing we have $\ln V(x)=-\alpha g(x)$.

Comment: we have used the fact that (39-41) are all the same equation, but in different functions of the same variables.

The proof of the Corollary is immediate because

$$
g(x+y)=g(x)+g(y)
$$

is the well-known Cauchy equation which, under strict monotonicity and onto $[0, \infty[$, has the solutions $g(x)=c x$. The rest follows from this and the Proposition 4 .

\section{References}

Abdellaoui, M., Bleichrodt, H., \& Paraschiv, C. (2007). Loss aversion under prospect theory: A parameterfree measurement. Management Science, 53, 1659-1674.

Aczél, J. (1966). Lectures on functional equations and their applications. New York: Academic Press.

Binmore, K. (2009). Interpersonal comparison of utility. In H. Kincaid \& D. Ross (Eds.), Oxford handbook of the philosophy of economic science (Chap. 20, pp. 540-559). Oxford University Press.

Elster, J., \& Roemer, J. (Eds.). (1991). Interpersonal comparisons of well-being. London: Cambridge University Press. 
Hammond, P. (1991). Interpersonal comparisons of utility: Why and how they are and should be made. In J. Elster \& J. Roemer (Eds.), Interpersonal comparisons of well-being. London: Cambridge University Press.

Harsanyi, J. (1977). Rational behavior and bargaining equilibrium in games and social situations. Cambridge: Cambridge University Press.

Hölder, O. (1901). Die Axiome der Quantität und die Lehre vom Mass. Berichte über die Verhandlungen der Königlich-Sächsischen Geselischaft der Wissenschaften zu Leipzig, Mathematisch-Physische Klasse, 53, $1-64$.

Keeney, R. L., \& Raiffa, H. (1993/1976). Decisions with multiple objectives. New York: Wiley.

Krantz, D. H., Luce, R. D., Suppes, P., \& Tversky, A. (1971). Foundations of measurement (Vol. I). New York: Academic Press [Reprinted by Dover Publications in 2007].

List, C. (2003). Are interpersonal comparisons of utility indeterminate? Erkenntnis, 58, 229-260.

Luce, R. D. (2000). Utility of gains and losses. Mahwah, NJ: Erlbaum.

Marley, A. A. J., \& Luce, R. D. (2002). A simple axiomatization of binary rank-dependent utility of gains (losses). Journal of Mathematical Psychology, 46, 40-55.

Narens, L., \& Luce, R. D. (1983). How we may have been misled into believing in the inter-personal comparability of utility. Theory and Decision, 15, 247-260.

Nozick, R. (1985). Interpersonal utility theory. Social Choice and Welfare, 2, 161-179 [Reprinted as Chapter 4 of Nozick, R. (1997). Socratic puzzles (pp. 85-109). Harvard University Press].

Ng, C. T., Luce, R. D., \& Marley, A. A. J. (2009). Utility of gambling under p-additive joint receipt and segregation or duplex decomposition. Journal of Mathematical Psychology (under review).

Robbins, L. (1938). Interpersonal comparisons of utility: A comment. The Economic Journal, 48, 635-641. 